\title{
ANGIOGRAFIA POR TOMOGRAFIA COMPUTADORIZADA DOS ANEURISMAS INTRACRANIANOS*
}

\author{
Vinícius Santos Laureano ${ }^{1}$, José Carlos Zirretta², Hilton Augusto Koch ${ }^{3}$
}

Resumo Este trabalho foi realizado para avaliar os achados da angiografia por tomografia computadorizada, comparativamente à angiografia por subtração digital, em relação aos aneurismas intracranianos, e a possibilidade da maior utilização da angiografia por tomografia computadorizada no Brasil. Foram analisados oito pacientes que apresentavam um total de sete aneurismas não tratados e um aneurisma tratado. Houve subseqüente correlação com outros trabalhos publicados na literatura médica. Os exames foram realizados em uma clínica e em dois hospitais privados, na cidade do Rio de Janeiro, RJ. Foi demonstrada boa correlação entre os métodos estudados, em relação ao diagnóstico dos aneurismas, no acompanhamento e avaliação pré-terapêutica dessas lesões. A angiografia por tomografia computadorizada foi um exame menos invasivo, de menor custo e maior acessibilidade. Concluiu-se que a angiografia por tomografia computadorizada é um exame que deve ser utilizado com maior freqüência para avaliação nesta enfermidade.

Unitermos: Aneurisma intracraniano. Tomografia computadorizada por raios X. Angiografia cerebral. Radiologia.

\begin{abstract}
Computed tomography angiography of intracranial aneurysms.
We conducted a study to assess and compare the findings of computed tomography angiography and digital subtraction angiography in patients with intracranial aneurysms, and to evaluate the possibility of a larger utilization of computed tomography angiography in Brazil. Eight patients were studied, 7 with nontreated and 1 with a treated aneurysm. The results were compared with those from the medical literature. Tests were performed at a private clinic and in two private hospitals in Rio de Janeiro, RJ, Brazil. There was good correlation between the two investigational methods regarding diagnosis, follow-up and pretreatment evaluation of these lesions. Computed tomography angiography showed to be less invasive, less costly and more accessible. We concluded that computed tomography angiography should be used more often in the evaluation of intracranial aneurysms.

Key words: Intracranial aneurysm. X-ray computed tomography. Cerebral angiography. Radiology.
\end{abstract}

\section{INTRODUÇÃO}

Recentemente, os aparelhos de tomografia computadorizada (TC) de última geração, também chamados helicoidais ou espirais, vêm permitindo exames ultra-rápidos, com cortes de espessura cada vez mais fina. A computação gráfica, mediante utilização destes dados, nos dá a possibilidade de fazer reconstruções multiplanares da anatomia, e mesmo tridimensionais. Os vasos sanguíneos podem ser destacados pelo uso do meio de contraste endovascu-

* Trabalho realizado na Clínica Labs Madureira e no Hospital Rio-Mar, Rio de Janeiro, RJ.

1. Radiologista Chefe do Serviço de Diagnóstico por Imagem do Hospital Quinta D'Or e da Clínica Labs Madureira.

2. Professor de Radiologia da Universidade do Estado do Rio de Janeiro e Neurorradiologista do Hospital Rio-Mar.

3. Professor Titular do Departamento de Radiologia do Hospital Universitário Clementino Fraga Filho da Universidade Federal do Rio de Janeiro.

Endereço para correspondência: Dr. Vinícius Santos Laureano. Rua Américo Brasiliense, 236, Madureira. Rio de Janeiro, RJ, 21351-060. E-mail: laureano @ cremerj.com.br

Recebido para publicação em 3/8/2001. Aceito, após revisão, em 24/10/2001. lar iodado. Novos programas de computação permitem, inclusive, simular um exame endoscópico, seja de vísceras ocas, vias aéreas ou mesmo de vasos sanguíneos. Todos estes novos fatos permitiram o desenvolvimento da angiografia por tomografia computadorizada (ATC).

A possibilidade de avaliar estruturas vasculares por um método menos invasivo que a angiografia por subtração digital (ASD) agrada aos médicos assistentes, e parece ser uma tendência pela maior rapidez do exame, menor risco para o paciente, custo reduzido e validade clínica, facilitando o diagnóstico e acompanhamento. Além disso, a TC também estuda outras doenças intracranianas que podem estar presentes, concomitantemente, em um paciente com vasculopatia cerebral. Podemos, então, avaliar a relação dos aneurismas intracranianos com as estruturas ósseas, o parênquima e demais estruturas encefálicas. O objetivo deste trabalho é avaliar a ATC helicoidal, por meio de imagens bidimensionais multiplanares e tridimensionais, comparativamente à ASD, no estudo de aneurismas intracranianos, no tocante aos seguintes itens: a) diagnóstico; $b$ ) orientação de tratamento; c) acompanhamento; d) utilização em larga escala.

\section{PACIENTES, MATERIAL E MÉTODO}

Foram estudados, por meio da ATC e da ASD, oito pacientes com diagnóstico prévio de aneurisma intracraniano obtido por TC, ressonância magnética (RM) ou ASD, no período de 4/6/1997 até 2/6/2000. Um dos pacientes já havia sido tratado, sendo o estudo realizado como pós-operatório. $\mathrm{O}$ espaço entre os dois tipos de exame para um mesmo paciente variou de quatro a nove meses.

Os exames foram feitos e analisados em uma clínica e em dois hospitais privados na cidade do Rio de Janeiro, RJ. Os exames de ATC foram realizados em aparelho 
ProSpeed fabricado pela GE Medical Systems, por meio de cortes helicoidais com $1 \mathrm{~mm}$ de espessura, com "pitch" de 1:1 para estudo de aneurismas e "pitch" de 2:1 para estudo das malformações arteriovenosas. O "pitch" maior permite estudo de uma região de interesse de maior extensão, apesar da pequena perda de qualidade da imagem. $\mathrm{O}$ espaço entre a reconstrução das imagens foi de $0,5 \mathrm{~mm}$ ( $50 \%$ da espessura de corte). Os cortes foram feitos durante a injeção do meio de contraste iodado $(300 \mathrm{mg} / \mathrm{ml})$ em uma veia antecubital, com volume total de $120 \mathrm{ml}$, por intermédio de bomba injetora Medrad MCT. O “scan delay” variou de 17 a 20 segundos, mais rápido se mais jovem o paciente. A velocidade de fluxo utilizada variou de 2,5 a 3,0 ml/s, maior quando a veia puncionada permitia o uso de agulha de maior calibre.

Utilizando-se um computador (estação de trabalho SUN) e programa específico (Advantage Windows, GE), os dados obtidos foram reconstruídos por um médico radiologista, obtendo-se imagens bidimensionais multiplanares ou imagens tridimensionais pelas técnicas MIP ("maximum intensity projection" — projeção de intensidade máxima) e SSD ("shaded surface display" - exposição de superfície sombreada).

A análise foi feita na estação de trabalho e posteriormente impressa em filme radiológico. O tempo para a realização do exame de ATC foi aproximadamente o mesmo empregado em um exame de TC de crânio, cerca de dez minutos. A edição e o processamento das imagens feitos por um médico radiologista treinado leva cerca de 30 a 60 minutos, período no qual é também realizada a análise dos casos.

O estudo por angiografia digital foi realizado em aparelhos Siemens (três pacientes) e General Electric (cinco pacientes) por um neurorradiologista experiente, mediante punção da artéria femoral e cateterização seletiva de vasos intracranianos.

As medidas dos aneurismas pela ASD foram baseadas nas medidas das artérias basilar ou carótida interna como referência de valor, e este valor é arbitrado pela média dos diâmetros encontrados em pacientes submetidos a angiografia cerebral sem doença vascular ou por análise dos diâmetros destes vasos em peças anatômicas.
Zubillaga et al. ${ }^{(\mathbf{1})}$, em trabalho de revisão, utilizam um valor de referência de cerca de 3,4 mm de diâmetro para os vasos citados.

Os exames de ATC e de ASD foram analisados separadamente e os seguintes dados foram obtidos: a) detecção; b) localização; c) tamanho; d) forma; e) colo; f) anatomia vascular de vizinhança; g) relação com outras estruturas intracranianas.

Os achados de um e outro método foram comparados, sempre colocando a angiografia digital como método padrão.

\section{RESULTADOS}

$\mathrm{Na}$ angiografia digital, em sete de oito pacientes foram encontradas formações aneurismáticas, sendo que cinco pacientes tinham um aneurisma e dois pacientes apresentavam dois aneurismas. Um dos pacientes tinha um aneurisma embolizado, sendo o exame realizado como pós-operatório. O número total de aneurismas evidenciados na ASD foi então de nove. A faixa etária dos pacientes variou de 43 a 68 anos. Dos oito pacientes, quatro $(50 \%)$ eram do sexo masculino e quatro $(50 \%)$ eram do sexo feminino.

A localização dos aneurismas e suas dimensões (Figuras 1 e 2) estão sumarizadas na Tabela 1. As dimensões dos aneurismas variaram de 3 a $62 \mathrm{~mm}$, com média de $12,9 \mathrm{~mm}$.

A ATC demonstrou os mesmos aneurismas que a angiografia digital. A localização dos aneurismas foi igual em relação à ASD. A dimensão dos aneurismas variou de 3 a $67 \mathrm{~mm}$, com média de 13,4 mm. Os dados estão sumarizados na Tabela 2 .

A anatomia vascular intracraniana foi bem avaliada em relação aos principais vasos, notadamente do polígono de Willis, e mesmo pequenos vasos puderam ser bem observados.

\section{DISCUSSÃO}

Com recentes e crescentes avanços tecnológicos, novas formas de diagnóstico por imagem têm sido desenvolvidas. Torna-se, então, indispensável definir, cientificamente, qual é o papel de cada método diagnóstico no que concerne não só à eficácia diagnóstica, como a outros vários parâmetros, inclusive custos.

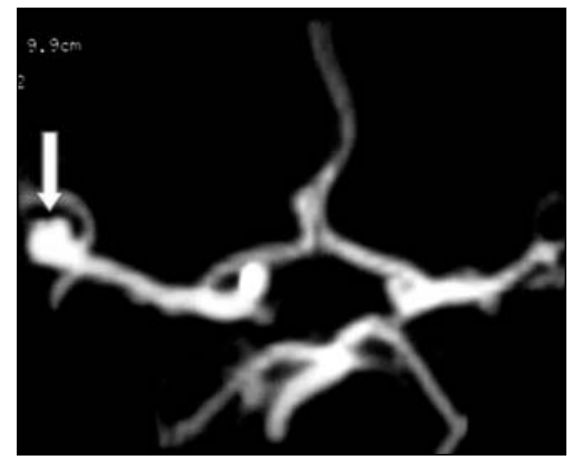

Figura 1. Reconstrução de aneurisma em MIP. Aneurisma sacular da bifurcação da artéria cerebral média direita (seta). Paciente 8.

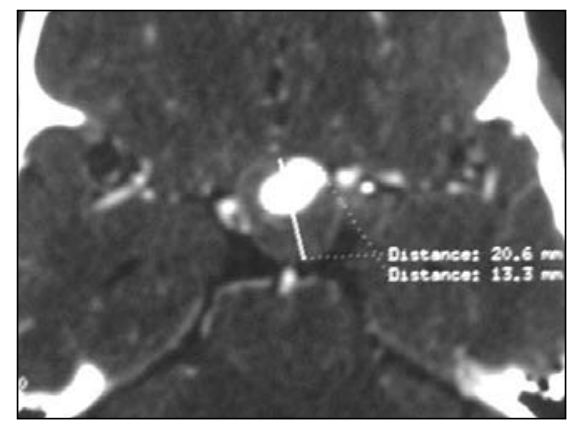

Figura 2. Angiografia por tomografia computadorizada. Aneurisma sacular parcialmente trombosado da artéria comunicante anterior. Paciente 3.

Vários trabalhos já foram realizados comparando a ATC e a angiografia digital em relação ao diagnóstico de aneurismas intracranianos, com dados que demonstram que a técnica menos invasiva é bastante promissora, com índices de sensibilidade comparáveis à $\mathrm{ASD}^{(2-7)}$. A Tabela 3 traz um resumo desses trabalhos.

Parece haver importante limitação, provavelmente a ser superada com o avanço tecnológico, sendo relacionada a aneurismas menores que $3 \mathrm{~mm}$, sendo $2 \mathrm{~mm}$, talvez, o limite inferior para a acuidade diagnóstica $^{(5,8)}$.

As imagens bidimensionais são evidentemente superiores para a avaliação de pequenos vasos, pois as reconstruções tridimensionais, sendo objeto de manipulação de dados, terminam por "amputá-los" ou excluí-los. Há que se fazer uma ressalva em relação à dificuldade de individualização dos vasos próximos à calota craniana, pela dificuldade de separá-los nas reconstruções tridimensionais.

É interessante, ainda, acrescentar que a maior demanda de tempo ocorre não na 
Tabela 1 Sumário dos achados nas angiografias por subtração digital em pacientes com aneurismas.

\begin{tabular}{|c|c|c|c|c|c|}
\hline $\begin{array}{l}\text { № do } \\
\text { exame }\end{array}$ & $\begin{array}{c}\text { № de } \\
\text { aneurismas }\end{array}$ & Local & Dimensões & Forma & Colo \\
\hline 1 & 1 & Artéria cerebral posterior esquerda & $10 \times 11 \times 10 \mathrm{~mm}$ & Sacular & $5 \mathrm{~mm}$ \\
\hline \multirow[t]{2}{*}{2} & \multirow[t]{2}{*}{2} & Artéria carótida esquerda segmento C6 & $14 \times 10 \times 10 \mathrm{~mm}$ & Sacular & $3 \mathrm{~mm}$ \\
\hline & & Artéria hipofisária superior direita & $8 \times 5 \times 6 \mathrm{~mm}$ & Sacular multilobulado & $1 \mathrm{~mm}$ \\
\hline 3 & 1 & Artéria comunicante anterior & $21 \times 22 \times 16 \mathrm{~mm}$ & Sacular parcialmente trombosado & $3 \mathrm{~mm}$ \\
\hline 4 & 1 & Artéria basilar & $23 \times 13 \times 62 \mathrm{~mm}$ & $\begin{array}{l}\text { Misto, sacular e fusiforme, parcialmente } \\
\text { trombosado }\end{array}$ & Não \\
\hline 5 & 1 & Artéria basilar & $18 \times 8 \times 16 \mathrm{~mm}$ & Sacular bilobulado & Não \\
\hline 6 & 1 & Artéria basilar & Embolizado & Embolizado & Embolizado \\
\hline \multirow[t]{2}{*}{7} & \multirow[t]{2}{*}{2} & Artéria pericalosa & $4 \times 4 \mathrm{~mm}$ & Sacular & Não \\
\hline & & Artéria cerebral média esquerda & $3 \times 3 \mathrm{~mm}$ & Sacular & Não \\
\hline 8 & 1 & Artéria cerebral média direita & $8 \times 4 \mathrm{~mm}$ & Sacular & $3 \mathrm{~mm}$ \\
\hline
\end{tabular}

Tabela 2 Sumário dos achados nas angiografias por tomografia computadorizada em pacientes com aneurismas.

\begin{tabular}{|c|c|c|c|c|c|}
\hline $\begin{array}{l}\text { № do } \\
\text { exame }\end{array}$ & $\begin{array}{c}\text { № de } \\
\text { aneurismas }\end{array}$ & Local & Dimensões & Forma & Colo \\
\hline 1 & 1 & Artéria cerebral posterior esquerda & $12,3 \times 10,0 \times 10,8 \mathrm{~mm}$ & Sacular & $6 \mathrm{~mm}$ \\
\hline \multirow[t]{2}{*}{2} & \multirow[t]{2}{*}{2} & Artéria carótida esquerda segmento C6 & $13,1 \times 9,7 \times 11,3 \mathrm{~mm}$ & Sacular & $4,5 \mathrm{~mm}$ \\
\hline & & Artéria hipofisária superior direita & $6,9 \times 5,2 \times 6,6 \mathrm{~mm}$ & Sacular multilobulado & $1,4 \mathrm{~mm}$ \\
\hline 3 & 1 & Artéria comunicante anterior & $23,0 \times 20,5 \times 18,4 \mathrm{~mm}$ & Sacular parcialmente trombosado & $2,2 \mathrm{~mm}$ \\
\hline 4 & 1 & Artéria basilar & $21,9 \times 14,8 \times 67,0 \mathrm{~mm}$ & $\begin{array}{l}\text { Misto, sacular e fusiforme, parcialmente } \\
\text { trombosado }\end{array}$ & Não \\
\hline 5 & 1 & Artéria basilar & $19,3 \times 8,0 \times 16,4 \mathrm{~mm}$ & Sacular bilobulado & Não \\
\hline 6 & 1 & Artéria basilar & Embolizado & Embolizado & Embolizado \\
\hline \multirow[t]{2}{*}{7} & \multirow[t]{2}{*}{2} & Artéria pericalosa & $4,2 \times 4,9 \mathrm{~mm}$ & Sacular & Não \\
\hline & & Artéria cerebral média esquerda & $3 \times 3 \mathrm{~mm}$ & Sacular & Não \\
\hline 8 & 1 & Artéria cerebral média direita & $7,0 \times 5,2 \mathrm{~mm}$ & Sacular & $2,2 \mathrm{~mm}$ \\
\hline
\end{tabular}

Tabela 3 Sensibilidade e especificidade no diagnóstico de aneurismas intracranianos nas angiografias por tomografia computadorizada.

\begin{tabular}{|c|c|c|c|c|}
\hline Autor & $\begin{array}{c}\text { № de } \\
\text { pacientes }\end{array}$ & $\begin{array}{c}\text { № de } \\
\text { aneurismas }\end{array}$ & $\begin{array}{c}\text { Sensibilidade } \\
(\%)\end{array}$ & $\begin{array}{c}\text { Especificidade } \\
(\%)\end{array}$ \\
\hline Schwartz et al. ${ }^{(9)}$ (1994) & 21 & 30 & 87 & 100 \\
\hline Alberico et al. ${ }^{(5)}(1995)$ & 68 & 24 & 96 & 100 \\
\hline Vieco et al. (1995)* & 22 & 30 & 87 & 94 \\
\hline Liang et al. (1995)* & 23 & 17 & 88 & 89 \\
\hline Wilms et al. ${ }^{(7)}$ (1996) & 10 & 14 & 100 & Não relatado \\
\hline Rieger et al. (1996)* & 32 & 9 & 100 & Não relatado \\
\hline Hope et al. ${ }^{(10)}(1996)$ & 80 & 94 & 90 & 50 \\
\hline Zouaoui et al. ${ }^{(3)}$ (1997) & 107 & 129 & 97 & 100 \\
\hline Lenhart et al.(11) (1997) & 53 & 51 & 98 & 100 \\
\hline Young et al..(4) (1998) & 66 & 55 & 95 & 74 \\
\hline
\end{tabular}

* Dados relatados no trabalho de Kuszyk et al. ${ }^{(2)}$.

realização do exame, mas no processamento das imagens na estação de trabalho ${ }^{(\mathbf{1 2}, 13)}$.

Os dados deste trabalho mostram boa correlação da ATC com a ASD, em relação aos dados pesquisados. Sendo a ATC um exame rápido, de menor custo e menos invasivo, a sua utilização pode ser recomendável. Porém, segundo Osborn ${ }^{(\mathbf{1 4})}$, embora as técnicas não-invasivas - ATC e angio- grafia por RM (ARM) - sejam freqüentemente efetivas em pacientes com aneurismas não rotos, a maioria dos neurocirurgiões ainda prefere uma combinação de TC sem contraste e ASD para avaliar pacientes com possível hemorragia subaracnóidea. Segundo Brown et al. ${ }^{\text {(15) }}$, o sucesso da ATC é evidenciado pela crescente aceitação como exame de rotina. Atualmente, os novos aparelhos de TC incorporam quase sempre a tecnologia helicoidal, além destes existirem em muito maior número do que os aparelhos de ASD. Estes fatos implicam que a não-utilização da ATC, sendo esta comprovada como excelente para diagnóstico de aneurismas intracranianos, é, pelo menos, um grande desperdício.

Como este trabalho relata a impressão sobre aneurismas não rotos, é preciso ressaltar que alguns artigos publicados demonstram que a ATC é um bom exame nos pacientes com hemorragia subaracnói$\mathrm{dea}^{(\mathbf{1 1}, \mathbf{1 6})}$, podendo, inclusive, em algumas circunstâncias, diagnosticar aneurismas quando a ASD for negativa ${ }^{(17)}$.

No que se refere ao custo, não temos dados nacionais, porém Harbaugh et al. ${ }^{(\mathbf{1 8})}$ obtiveram e compararam os custos envolvidos (material, taxas hospitalares, custos profissionais) da ATC, ARM e ASD em New Hampshire, EUA, e demonstraram que entre 1992 e 1993 o custo médio de uma ATC era cerca de $31 \%$ de uma ASD e $72 \%$ de uma ARM. 
Somado a isto, pesa a favor da ATC a possibilidade da avaliação das estruturas encefálicas e da existência de alterações intracranianas concomitantes, o que é de bastante auxílio para o planejamento do tratamento, seja clínico, cirúrgico ou endovascular $^{(16,18-22)}$.

É conhecida uma "verdadeira revolução" ocorrida na neurologia após o desenvolvimento da TC, e, apesar do surgimento da RM, a TC ainda é um método reconhecidamente eficaz para estudo das estruturas encefálicas, sendo superior à RM na avaliação de calcificações, da cortical óssea e de pequenas estruturas ósseas. Além disso, a possibilidade de utilização de reconstruções bi e tridimensionais melhora a observação da anatomia das estruturas intracranianas.

Deve-se lembrar que, quando se descreve a ASD como técnica invasiva, isto se refere ao fato de, apesar da utilização de técnicas modernas e meticulosas, mesmo em centros de referência, as complicações serem possíveis, inclusive nas mãos do mais habilidoso angiografista. Em pacientes com hemorragia subaracnóidea o risco pode ser maior, considerando-se o aparecimento ou agravamento de angioespasmo, bem como em pacientes idosos, com doença cérebro-vascular difusa, com hipertensão arterial não controlada ou com ataques isquêmicos transitórios freqüentes ${ }^{(\mathbf{1 8})}$.

Neste estudo não foi feita a comparação com a ARM, mas é conveniente citá-la. A ARM também é um método não-invasivo, e segundo Katz et al. ${ }^{(23)}$ e Schwartz et al. ${ }^{(\mathbf{9})}$, a ARM e a ATC são métodos comparáveis em termos de sensibilidade no estudo da vascularização intracraniana. Porém, segundo Tacke et al. ${ }^{(\mathbf{1 6})}$, em seu trabalho de 1997, embora a imagem multidimensional por RM tenha trazido enorme melhora na orientação anatômica, a transferência da informação adquirida ao sítio anatômico, como necessário em uma cirurgia de base de crânio, permanece difícil. Já a TC helicoidal com reconstrução tridimensional de superfície é uma técnica moderna que claramente melhora a compreensão espacial das alterações ósseas ou a relação entre tumores ou aneurismas e a base do crânio. Esses autores concluíram que exames de ATC em aneurismas em topografia anterior, média ou posterior na base do crânio foram classificados como de ajuda ou significantemente informativos na sua maioria, na avaliação pré e pós-operatória, tendo inclusive, subjetivamente, avaliado como de muita ajuda em mais da metade dos casos. Ademais, conforme descrito anteriormente, o custo de um exame de RM é maior, além do fato de haver menor disponibilidade destes aparelhos.

Segundo Wilms et al. ${ }^{(7)}$ e Schwartz ${ }^{(24)}$, em pacientes com alta velocidade de fluxo sanguíneo cerebral o realce precoce do seio cavernoso pode obscurecer a carótida intracavernosa na ATC (deve-se ressaltar, apesar de não referido por este autor, que este problema pode ser parcialmente contornado com reconstruções multiplanares bidimensionais, reconstruções tridimensionais e correta manipulação da janela) e o uso do meio de contraste iodado é uma desvantagem (alergia e insuficiência renal). Porém, a ATC tem várias vantagens definitivas sobre a ARM. A ATC é mais bem tolerada por claustrofóbicos, há menos artefatos de movimento pela rapidez do exame, a imagem não é prejudicada em pacien- tes com insuficiência cardíaca, como pode acontecer na ARM, não há artefatos de fluxo em grandes aneurismas e os trombos e calcificações são melhor observados.

Um outro dado que parece importante é a confiabilidade das medidas dos aneurismas, visto que pela ATC podem ser medidos de parede externa a parede externa, incluindo trombos e espessamentos parietais, o que não pode ser feito pela ASD, bem como as medidas realizadas por ASD são, em geral, menos precisas.

Os parâmetros técnicos utilizados para a realização dos exames foram escolhidos após comparação com a literatura, conforme está descrito na Tabela 4. Utilizamos o corte mais fino, no tempo mais curto possível que permitisse cobrir a área de estudo adequada, o menor "pitch" e reconstrução das imagens com $50 \%$ da espessura de corte. Estes parâmetros buscaram a melhor qualidade de imagem possível ${ }^{(\mathbf{1 2})}$.

Houve alguma dificuldade em se conseguir um maior número de casos para este trabalho, pelo fato de este exame ainda não estar bem difundido no meio médico, além

Tabela 4 Revisão da literatura dos parâmetros técnicos da angiografia por tomografia computadorizada do crânio.

\begin{tabular}{|c|c|c|c|c|c|}
\hline Autor & $\begin{array}{l}\text { Colimação } \\
\qquad(\mathrm{mm})\end{array}$ & "Pitch" & $\begin{array}{c}\text { Volume de } \\
\text { contraste } \\
\text { (ml) }\end{array}$ & $\begin{array}{c}\text { Velocidade } \\
\text { de infusão } \\
\text { (ml/s) }\end{array}$ & $\begin{array}{l}\text { "Delay" } \\
\text { (s) }\end{array}$ \\
\hline Napel et al. (1992)* & 1 & 1 & 60 & 3 & 18 \\
\hline Dillon et al. (1993)* & 3 & 1 & $100-150$ & $1,5-2$ & $30-40$ \\
\hline Kalender et al. (1994)* & $1-2$ & 1 & $120-150$ & $3-4$ & 20 \\
\hline Görzer et al. (1994)* & 3 & 1 & 100 & 4 & 16 \\
\hline Rieger et al. $(1994)^{\S}$ & 1 & 1 & 80 & Não relatado & $14-17$ \\
\hline Schwartz $^{(24)}(1994)$ & 2 & 1 & 75 & 2,5 & 20 \\
\hline Tsuchiya et al. $(1994)^{\S}$ & 2 & 1 & 85 & 2 & 25 \\
\hline Dorsch et al. ${ }^{(6)}(1995)$ & 1 & 1 & 120 & 4 & 20 \\
\hline Vieco et al. $(1995)^{\S}$ & 1 & 1 & 100 & 2 & $20-40$ \\
\hline Zeman et al. $(1995)^{\S}$ & 1 & 1 & 100 & 2 & 30 \\
\hline Liang et al. (1995)* & 1 & 1 & 135 & 3 & 20 \\
\hline Rubin et al. (1995)* & Não relatado & Não relatado & 90 & 3 & $8-20$ \\
\hline Katz et al. (1995)* & 1 & 1,3 & 90 & 3 & Individual \\
\hline Alberico et al. ${ }^{(5)}$ (1995) & 1 & 1 & 80 & 3 & 15 \\
\hline Hsiang et al. ${ }^{(25)}(1996)$ & 1 & 1 & 135 & 3 & 20 \\
\hline Rieger et al. (1996)* & 1 & 1 & $60-80$ & 4 & $14-17$ \\
\hline Wilms et al..(7) (1996) & Não relatado & Não relatado & 100 & $3-3,5$ & 20 \\
\hline Dillon et al. $(1996)^{\S}$ & 1 & 1 & $87,5-140$ & $2,5-3,5$ & Não relatado \\
\hline Koch et al. $(1997)^{\S}$ & 1 & $1-1,5$ & $120-150$ & $3-3,5$ & $17-22$ \\
\hline Zouaoui et al. ${ }^{(3)}$ (1997) & 1 & 1 & 140 & 2,5 & $20-30$ \\
\hline Strayle-Batra et al. ${ }^{(26)}$ (1998) & 1 & 1,5 & $100-130$ & $2-2,5$ & $15-25$ \\
\hline Young et al. ${ }^{(4)}(1998)$ & 1 & 1 & Não relatado & 4 & 12 \\
\hline Kuszyk et al. ${ }^{(2)}(1998)$ & $1-2$ & 1,5 & 120 & 3 & 15 \\
\hline
\end{tabular}

* Dados relatados no trabalho de Strayle-Batra et al. ${ }^{(26)}{ }^{\S}$ Dados relatados no trabalho de Koch et al. ${ }^{(27)}$. 
de não haver um centro de referência para drenagem de pacientes com doença cérebro-vascular. Os hospitais universitários e públicos, locais mais adequados ao atendimento de urgência e ao estudo acadêmico, têm carência de tecnologia de ponta.

Deve-se lembrar que, mesmo quando não se dispõe da tecnologia helicoidal, a ATC pode ser realizada, porém em locais onde a região anatômica a ser estudada não seja extensa, o que se enquadra perfeitamente na "altura" do polígono de Willis ${ }^{(\mathbf{1 5})}$.

A ATC poderia ser o exame eleito como preferencial para a varredura de aneurismas intracranianos em pacientes nos quais esta pesquisa seja necessária e no seguimento pré e pós-tratamento destas alterações. Também deveria ser o primeiro exame em pacientes com hemorragia. Estas afirmações se baseiam nos seguintes fatos: a acurácia do método; o custo mais acessível que a ASD e a ARM; o maior número de aparelhos disponíveis; a crescente substituição dos aparelhos de TC convencional pela tecnologia espiral.

A ARM poderia ser a melhor opção em pacientes comprovadamente com alergia ao meio de contraste iodado, com insuficiência renal ou em casos de gravidez, lembrando-se que a alergia não é contra-indicação absoluta, pois pode ser feita a dessensibilização, sendo então o exame realizado em ambiente hospitalar com acompanhamento de anestesista.

A ASD permaneceria como o exame de maior confiabilidade, sendo realizada em casos de dúvidas, como nos casos de ATC negativas, quando a possibilidade do diagnóstico de aneurisma intracraniano for alta, como pré-operatório ou quando a opção for o tratamento endovascular.

É importante citar que, dos artigos revisados, todos foram favoráveis à utilização da ATC no diagnóstico dos aneurismas intracranianos. E, ainda mais, a experiência com o uso da ATC com TC helicoidal já se faz desde 1992, sendo um tempo bastante razoável para se conhecer a eficiência do método.

O reconhecimento da conveniência da utilização da ATC como técnica diagnóstica eficaz poderia inclusive colaborar para um melhor tratamento, e mesmo para evitar uma hemorragia fatal, como no caso ilustrativo relatado a seguir.

\section{Caso ilustrativo}

Paciente de 66 anos de idade, sexo masculino, hipertenso controlado, internado no dia 16/5/2000 em um hospital universitário da rede pública, queixava-se de cefaléia intensa de início súbito, vômitos em jato e apresentava rigidez de nuca ao exame físico. Foi submetido a TC convencional contrastada, que revelou a existência de aneurisma no polígono de Willis anterior à esquerda, mas não evidenciou sinais de hemorragia subaracnóidea na fase sem contraste. Na ocasião, o neurorradiologista do hospital alertou para a possibilidade de a cefaléia ter tido origem em pequeno sangramento sem repercussão na TC. Foi feita então punção lombar, que confirmou a hemorragia subaracnóidea.

Por impossibilidade da realização de ASD (inexistência do equipamento), o paciente foi enviado a uma clínica particular, no dia 25/5/2000, para realização de exame de ATC, que demonstrou aneurisma localizado no segmento comunicante (C7) da carótida intracraniana esquerda, lobulado, medindo $18 \mathrm{~mm}$ no maior diâmetro (Figura 3). A artéria comunicante posterior emergia do aneurisma e a artéria cerebral posterior tinha origem fetal. O colo do aneurisma media cerca de $4 \mathrm{~mm}$. Optou-se por realizar uma ASD, exame mais caro e com menor número de aparelhos para sua realização. O exame foi marcado em uma clínica privada, sendo que o paciente, infortunadamente, faleceu dois dias antes, vítima de nova e volumosa hemorragia.

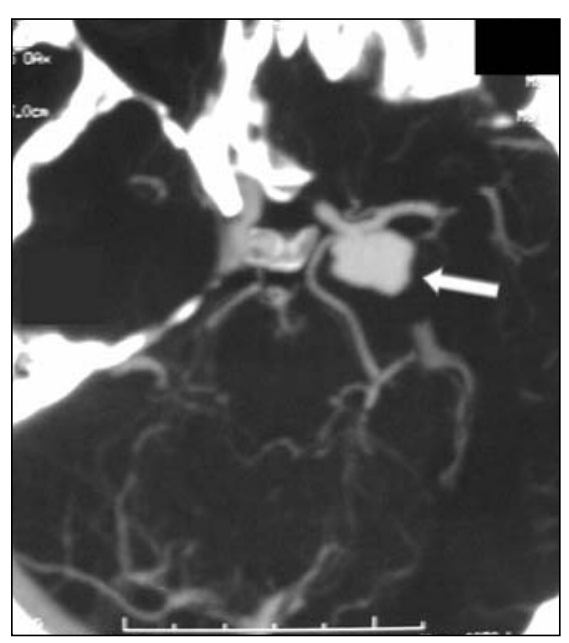

Figura 3. Aneurisma do caso ilustrativo, localizado no segmento C7 da artéria carótida intracraniana esquerda (seta).
Este paciente do caso ilustrativo não entrou na casuística do trabalho por não haver ASD comparativa. Porém, melhor seria se tivesse sido operado apenas de posse da ATC, segundo as equipes neurocirúrgica e neurorradiológica.

\section{CONCLUSÕES}

A ATC é um exame comparável à ASD na avaliação diagnóstica dos aneurismas intracranianos.

A ATC pode cumprir papel importante na orientação terapêutica dos aneurismas intracranianos, tendo vantagem, comparativamente à ASD, no que concerne ao estudo bi e tridimensional da relação da lesão com as estruturas encefálicas e pela avaliação de lesões encefálicas concomitantes.

A ATC pode ser utilizada no acompanhamento dos aneurismas intracranianos, com algumas vantagens em relação à ASD por ter custo menor, menor índice de complicações e ser mais acessível.

A ATC é exame que pode ser realizado em larga escala por ter, comparativamente, custo menor do que a ASD, além da crescente aquisição e evolução dos aparelhos de TC que são capazes de realizar o exame.

\section{REFERÊNCIAS}

1. Zubillaga AF, Guglielmi G, Viñuela F, Duckwiler GR. Endovascular occlusion of intracranial aneurysms with electrically detachable coils: correlation of aneurysm neck size and treatment results. AJNR 1994;15:815-20.

2. Kuszyk BS, Beauchamp NJ Jr, Fishman EK. Neurovascular applications of CT angiography. Semin Ultrasound CT MR 1998;19:394-404.

3. Zouaoui A, Sahel M, Marro B, et al. Three-dimensional computed tomographic angiography in detection of cerebral aneurysms in acute subarachnoid hemorrhage. Neurosurgery 1997;41: 125-30.

4. Young N, Dorsch NW, Kingston RJ, Soo MY, Robinson A. Spiral CT scanning in the detection and evaluation of aneurysms of the circle of Willis. Surg Neurol 1998;50:50-61.

5. Alberico RA, Patel M, Casey S, Jacobs B, Maguire W, Decker R. Evaluation of the circle of Willis with three-dimensional CT angiography in patients with suspected intracranial aneurysms. AJNR 1995;16:1571-8.

6. Dorsch NW, Young N, Kingston RJ, Compton JS. Early experience with spiral CT in the diagnosis of intracranial aneurysms. Neurosurgery 1995;36:230-6.

7. Wilms G, Guffens M, Gryspeerdt S, et al. Spiral CT of intracranial aneurysms: correlation with digital subtraction and magnetic resonance angiography. Neurosurgery 1996;38 Suppl 1:S20-5. 
8. Nakajima Y, Yoshimine T, Yoshida H, et al. Computerized tomography angiography of ruptured cerebral aneurysms: factors affecting time to maximum contrast concentration. J Neurosurg 1998; 88:663-9.

9. Schwartz RB, Tice HM, Hooten SM, Hsu L, Stieg PE. Evaluation of cerebral aneurysms with helical CT: correlation with conventional angiography and MR angiography. Radiology 1994;192: 717-22.

10. Hope JK, Wilson JL, Thomson FJ. Three-dimensional CT angiography in the detection and characterization of intracranial berry aneurysms. AJNR 1996;17:439-45.

11. Lenhart M, Bretschneider T, Gmeinwieser J, Ullrich OW, Schlaier J, Feuerbach S. Cerebral CT angiography in the diagnosis of acute subarachnoid hemorrhage. Acta Radiol 1997;38:791-6.

12. Kuszyk BS, Fishman EK. Technical aspects of CT angiography. Semin Ultrasound CT MR 1998;19:383-93.

13. Huang PL, Bahia PRV, Bayão JGA, Corrêa EB, Corrêa JM. Angiotomografia computadorizada helicoidal do crânio. Radiol Bras 1997;30:30916.

14. Osborn AG. Diagnostic cerebral angiography. 2nd ed. Philadelphia: Lippincott Williams \& Wilkins, 1999.
15. Brown JH, Lustrin ES, Lev MH, Taveras JM. CT angiography of the circle of Willis: is spiral technology always necessary? AJNR 1997;18:17947.

16. Tacke J, Klein HM, Bertalanffy $\mathrm{H}$, et al. Clinical significance of threee-dimensional helical CT in neurosurgery. Minim Invasive Neurosurg 1997; 40:30-5.

17. Hashimoto H, Iida J, Hironaka Y, Okada M, Sakaki T. Use of spiral computerized tomography angiography in patients with subarachnoid hemorrhage in whom subtraction angiography did not reveal cerebral aneurysms. J Neurosurg 2000;92: 278-83.

18. Harbaugh RE, Schlusselberg DS, Jeffrery R, et al. Three-dimensional computed tomographic angiography in the preoperative evaluation of cerebrovascular lesions. Neurosurgery 1995;36: 320-7.

19. Bartylla K, Hagen T, Waziri A, Moringlane JR. CT-Angiographie in der Planung stereotaktischer Biopsien. Neuroradiologie 1996;36:867-71.

20. Vieco PT. CT angiography of the intracranial circulation. Neuroimaging Clin N Am 1998;8:57792.

21. Moringlane JR, Bartylla K, Hagen T, Waziri A. Stereotactic neurosurgery planning with 3 -D spiral CT-angiography. Minim Invasive Neurosurg
1997;40:83-6.

22. Tanaka H, Numaguchi Y, Konno S, Shrier DA, Shibata DK, Patel U. Initial experience with helical CT and 3D reconstruction in therapeutic planning of cerebral AVMs. Comparison with 3D time-of-flight MRA and digital subtraction angiography. J Comput Assist Tomogr 1997;21: 811-7.

23. Katz DA, Marks MP, Napel SA, Bracci PM, Roberts SL. Circle of Willis: evaluation with spiral CT angiography, MR angiography, and conventional angiography. Radiology 1995;195:445-9.

24. Schwartz RB. Neuroradiological applications of spiral CT. Semin Ultrasound CT MR 1994;15 139-47.

25. Hsiang JN, Liang EY, Lam JM, Zhu XL, Poon WS. The role of computed tomographic angiography in the diagnosis of intracranial aneurysms and emergent aneurysm clipping. Neurosurgery 1996;38:481-7.

26. Strayle-Batra M, Skalej M, Wakhloo AK, Ernemann U, Klier R, Voigt K. Three-dimensional spiral CT angiography in the detection of cerebral aneurysm. Acta Radiol 1998;39:233-8.

27. Koch C, Grzyska U, Probst EN, et al. Die CTAngiographie zur diagnostischen Beurteilung intrakranieller Gef assaneurysmen. Wien Med Wochenschr 1997;147:163-71. 